\title{
3D convolutional GAN for fast simulation
}

\author{
Sofia Vallecorsa ${ }^{1,2, *}$, Federico Carminati ${ }^{1, * *}$, and Gulrukh Khattak $^{1,3, * * *}$ \\ ${ }^{1}$ CERN, Switzerland \\ ${ }^{2}$ Gangneung-Wonju National University, Korea \\ ${ }^{3}$ University of Peshawar, Pakistan
}

\begin{abstract}
Machine Learning techniques have been used in different applications by the HEP community: in this talk, we discuss the case of detector simulation. The need for simulated events, expected in the future for LHC experiments and their High Luminosity upgrades, is increasing dramatically and requires new fast simulation solutions. We describe an R\&D activity aimed at providing a configurable tool capable of training a neural network to reproduce the detector response and speed-up standard Monte Carlo simulation. This represents a generic approach in the sense that such a network could be designed and trained to simulate any kind of detector and, eventually, the whole data processing chain in order to get, directly in one step, the final reconstructed quantities, in just a small fraction of time. We present the first application of three-dimensional convolutional Generative Adversarial Networks to the simulation of high granularity electromagnetic calorimeters. We describe detailed validation studies comparing our results to Geant4 Monte Carlo simulation. Finally we show how this tool could be generalized to describe a whole class of calorimeters, opening the way to a generic machine learning based fast simulation approach.
\end{abstract}

\section{Introduction}

The future High Luminosity LHC phase will be very demanding in terms of computing resources, because of the amount and complexity of data that will be collected, stored and analysed [1]: correspondingly, the need for simulated data is expected to grow by one order of magnitude. For this reason, High Energy Physics (HEP) software and, in particular, Monte Carlo-based simulation, are going through an important phase of restructuring and optimisation for new computing architectures. In order to increase the performance of simulation applications, new methods and algorithms for fast simulation are being studied. Fast simulation approaches are already being used to reduce computation time: they are typically parametrized on physics quantities previously calculated or measured, trading-off some performance, in terms of physics accuracy, for speed. These solutions suffer from being specific to individual experiments and detectors and they can reach different levels of accuracy and speed.

\footnotetext{
*e-mail: sofia.vallecorsa@cern.ch

**e-mail: federico.carminati@cern.ch

***e-mail: gul.rukh.khattak@cern.ch
} 
Our proposal is to leverage state of the art deep learning algorithms to design a generic, fast simulation tool. The idea is to treat simulation as a black-box and replace detailed Monte Carlo with a deep neural network (DNN) that can be trained on a range of particle types, momenta, positions and detector configurations. In fact, the recent developments in deep learning provide a very promising avenue to replace complex algorithms with a suitably complex deep neural network that is able to reproduce the results of the former at a much higher speed. Generative models, like Generative Adversarial Networks (GANs) [2], are particularly suited to replace Monte Carlo simulation: their key feature is the ability to learn the underlying distribution and relevant features from the training dataset and reproduce new realistic samples according to the features they have learned.

This paper describes the first steps towards an automated tool to configure and train an optimised DNN according to the specific detector to simulate. We report on results of the initial prototyping and validation phase, focused on a specific use case, an example of future fine grained digital calorimeters. After a short introduction about related work in section 2, sections 3 and 4 introduce the calorimeter data and describe the GAN model architecture. Selected examples of physics validation are summarised in section 5. Section 6 presents an overview of computing performance for the inference and training processes, while section 7 concludes with a summary and an outlook on our future plans.

\section{Related Work}

GANs were introduced in 2014 [2]: they consist of two networks, a generator and a discriminator, trained in an adversarial manner. The original formulation defined the training problem as a min-max game with the generator and the discriminator competing against each other. The generator network is trained to generate realistic sample starting from random noise, while the discriminator network analyses the generator output and tags it as real (belonging to the original dataset) or fake. Assuming a large enough training sample, a unique solution to the problem exists with the generator covering the entire data distribution and the discriminator estimating a 50\% probability of being real for both real and generated images [2]. Many different GAN applications have been developed in the past past few years, in particular in the field of image processing [3], [4]. As we will see, the output of HEP detectors can be also be interpreted as an image, thus these same techniques can be employed to simulate their output. The LAGAN or (Location Aware GAN) [5] and CaloGAN [6] were among the first examples interpreting calorimeter simulation in these terms: CaloGAN, for example, generated the ATLAS Liquid Argon calorimeter output as a sequence of three two-dimensional images.

The present work is intended to move further along this direction, simulating threedimensional calorimeter showers as a whole.

\section{Calorimeter Data}

The training data set was produced in the context of the CLIC detector design [7]. It consists of energy showers generated with the Geant4 software [8] inside the CLIC electromagnetic and hadronic calorimeters detector prototype [7]. Our current study focuses on the electromagnetic calorimeter (ECAL). This is an example of next generation highly granular calorimeters and therefore it represents a demanding use case simulation will have to face during High Luminosity LHC runs. The data set was produced in an effort to provide the HEP community with a common benchmark to perform tests, development and optimization of different machine learning techniques [9]. The ECAL is a sampling calorimeter consisting 
of 25 layers of Tungsten absorber with silicon sensors in between them. The first 17 absorber layers have a thickness of $2.4 \mathrm{~mm}$, while the other 8 layers are twice as thick. Each plane of silicon sensor is $0.5 \mathrm{~mm}$ thick and it is segmented in $(5.1 \mathrm{~mm} \times 5.1 \mathrm{~mm})$ cells. The inner radius of the calorimeter is $1.5 \mathrm{~m}$.

An individual entry in the data set represents the energy deposited in the calorimeter cells, by the particle shower propagating through the detector. Each cell is characterised by the energy recorded in it and three indices (iX, iY, iZ), identifying its position. The $z$ axis is chosen along the direction of the incoming electron, while $x$ and $y$ are on the transverse plane, perpendicular to $z$. For each of the 25 ECAL sensor planes, a $25 \times 25$ array of cells is defined around the energy shower barycentre. The information stored for the ECAL shower is then $\mathrm{E}(\mathrm{iX}, \mathrm{iY}, \mathrm{iZ})$ for each of the $25 \times 25 \times 25$ cells. Each dataset entry also includes the information on the nature of the incoming particle and its energy. About $1 \mathrm{M}$ showers have been generated for each particle type (electrons, photons and pions). The energy of the incoming particle is sampled from a uniform $[10-500] \mathrm{GeV}$ spectrum. The baseline training dataset is composed of particles entering the calorimeter along a direction orthogonal to its surface (the $z$ axis). A second dataset was generated including particles with an incident angle $60^{\circ}<\theta<120^{\circ}$. The corresponding 3D images are $4 \mathrm{x}$ larger in order to entirely contain the energy deposits: they result in $51 \times 51 \times 25$ pixels. We use this "angle-" dataset to perform generalisation studies as explained in section 5 .

\section{The 3DGAN model architecture}

Our 3DGAN model is based on three-dimensional convolutions in order to capture the whole shower development along the three spatial dimensions. Both the generator and discriminator networks contain four convolutional layers [10]. The generator uses as input a vector of random numbers (the latent vector) generated according to normal distributions. The latent vector is multiplied by the input particle energy, a continuous parameter that effectively "conditions" the training [11]: in an unconditioned generative model, there is no control on the modes being generated. However, by conditioning on additional information it is possible to direct the data generation process and isntruct the Generator to create, for example, energy showers corresponding to a specific particle energy. Convolutions are chained to up-sampling layers in order to produce images of increasing size. Batch normalization layers for regularization and leaky rectified linear units activation functions are used to take into account the level of sparsity of the input images. The generator output consists of a $25 \times 25 \times 25$ ECAL image.

The discriminator takes as input the generated image. Its structure is very similar to the generator. In addition, it includes pooling layers with the dual purpose of down-sampling and regularization. A $2 \%$ drop out provides additional regularization against over-fitting. A set of three dense layers maps the flattened discriminator network response to three different outputs.

Loosely following the strategy of auxiliary classifier GANs [12], indicating that the introduction of labels provides faster convergence and stability, we assign to the discriminator two additional regression tasks: an estimation of the incoming particle energy $\left(E_{p}\right)$ and of the total energy measured by the calorimeter $\left(E_{c a l}\right)$, corresponding to the sum of all the energy depositions in the cells of the image.

The adversarial training uses a loss function based on the probability of the discriminator correctly differentiating between real and fake images (binary cross entropy). The generator in turn uses the inverse of the discriminator loss as the cost to minimize. The absolute mean percentage error is used to calculate the loss terms corresponding to the additional regression tasks performed by the discriminator: the incoming particle energy and total energy 

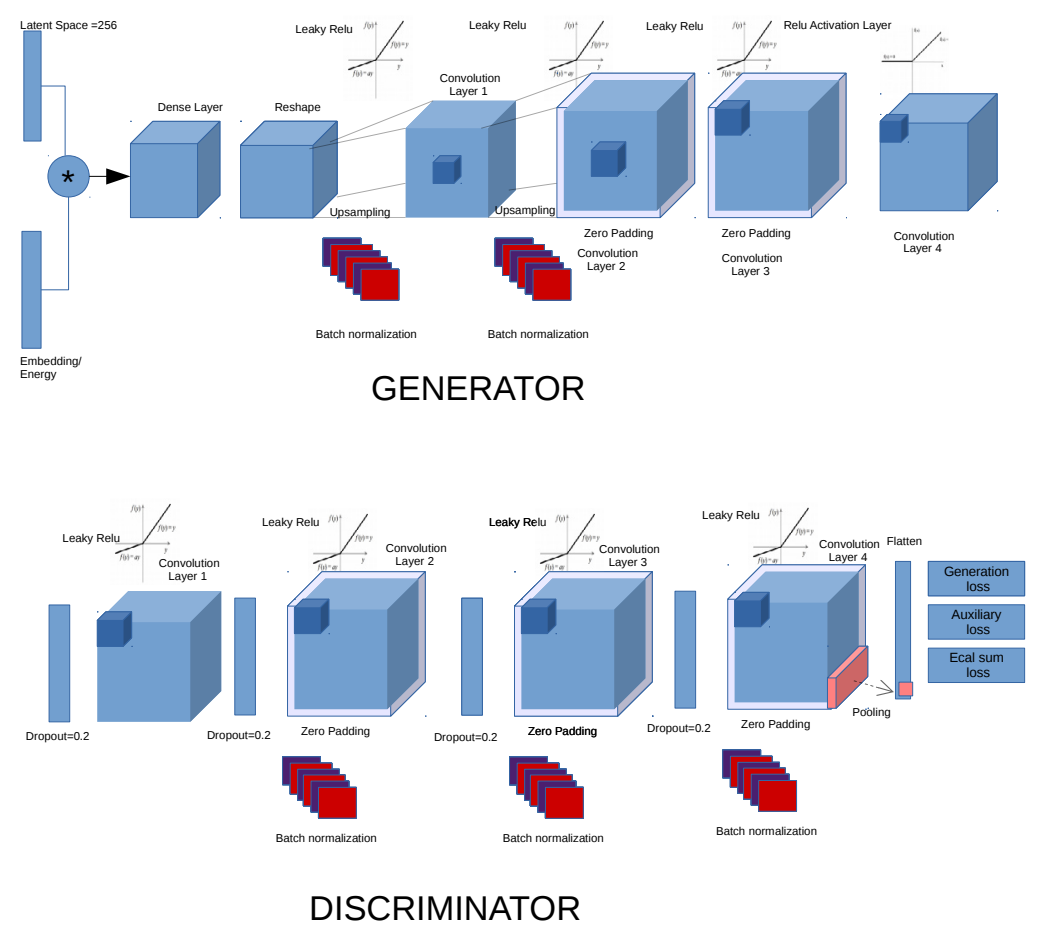

Figure 1. The three dimensional GAN model showing the generator and discriminator network architectures

deposited in the detector. Weights are employed to balance the contributions of the three individual terms to the total loss. The generator and discriminator cross entropy loss functions evolution is shown for a typical training run in Figure 2: the losses change smoothly during the training process and asymptotically approach the expected value describing the Nash game equilibrium [2]. Figure 2 also shows the real/fake probability, as estimated by the discriminator, for a real image (in blue) and a generated one (orange). The optimal result in the "training game" should peak around 0.5 showing that the discriminator can no longer distinguish generated images from the original ones.

The RMSProp [13] optimizer is used, with a learning rate of $10^{-3}$. We train the GAN models on 200000 electrons generated with a uniform energy between 10 and $500 \mathrm{GeV}$.

\section{Physics validation}

To fully validate GAN results in terms of physics we compare to standard Monte Carlo simulation based on Geant4. We have studied both low level variables describing the calorimeter energy response, i.e. the single cell energy distributions, and more complicated features such as average profiles of the electromagnetic showers along the three calorimeter axes. Figure 3 shows the shower profile along the $x$ and $z$ axes for $300 \mathrm{GeV}$ electrons: the distributions in $x$ and $y$ are similar so only the $x$ profile is presented. Figure 4 shows the longitudinal profile for different electron energies. The GAN prediction (red) is compared to the corresponding Geant4 simulation (blue). Figures 3 and 4 show that 3DGAN is capable of correctly repro- 

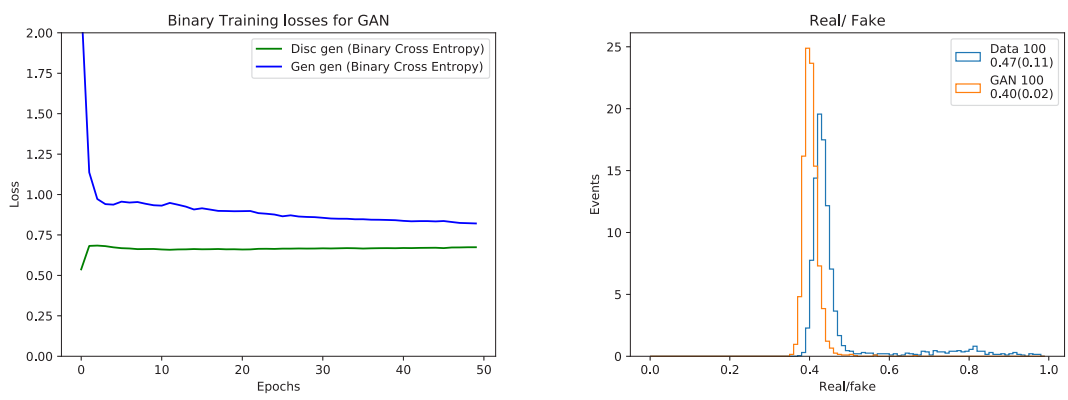

Figure 2. (left) Binary cross-entropy loss function for the generator and discriminator networks. (right) The discriminator real/fake probability for a real image (in blue) and a generated one (orange). The optimal result should peak around 0.5 showing that the discriminator can no longer distinguish the generated images from the original ones.
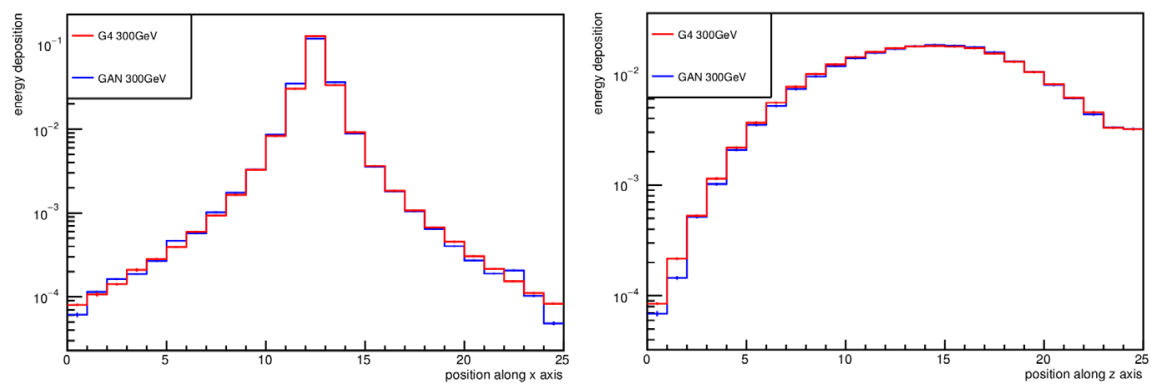

Figure 3. Transverse energy profile along $x$ (left) and longitudinal energy profile along $\mathrm{z}$ (right) for 300 $\mathrm{GeV}$ electrons. Geant 4 prediction is shown in blue and the GAN's in red. Units are GeV/50.

ducing the different profiles over a large energy range. The same agreement can be seen in Figure 5: it represents the calorimeter response in terms of deposited energy (calorimeter sampling fraction) for electrons and neutral pions. The network performance over this large energy range is remarkably good considered that no effort was made to optimise the network architecture for different energy values. Convolutional layers are sensitive to the spatial shape of the energy showers, which changes according to the primary particle energy, while the architecture parameters were optimised to reproduce at best particles with energies around the central region of the spectrum. The agreement in the case of neutral pions slightly deteriorates: we observed that this behaviour is consistent with the slightly smaller average energy depositions with respect to the electron sample.

As a first attempt to generalisation we tested our approach on the simulation of energy showers generated by electrons entering the calorimeter under a certain angle $\theta$, with respect to the orthogonal direction ("angle" dataset described in section 3). We made as little modifications as possible to the network architecture and training process: we increased the input image size along the $x$ and $y$ axes and adjusted the generator filter and upsampling factors in order to obtain an output image with the expected dimensions; finally we have conditioned the training using the input $\theta$ following the same procedure adopted of the primary energy [10]. 

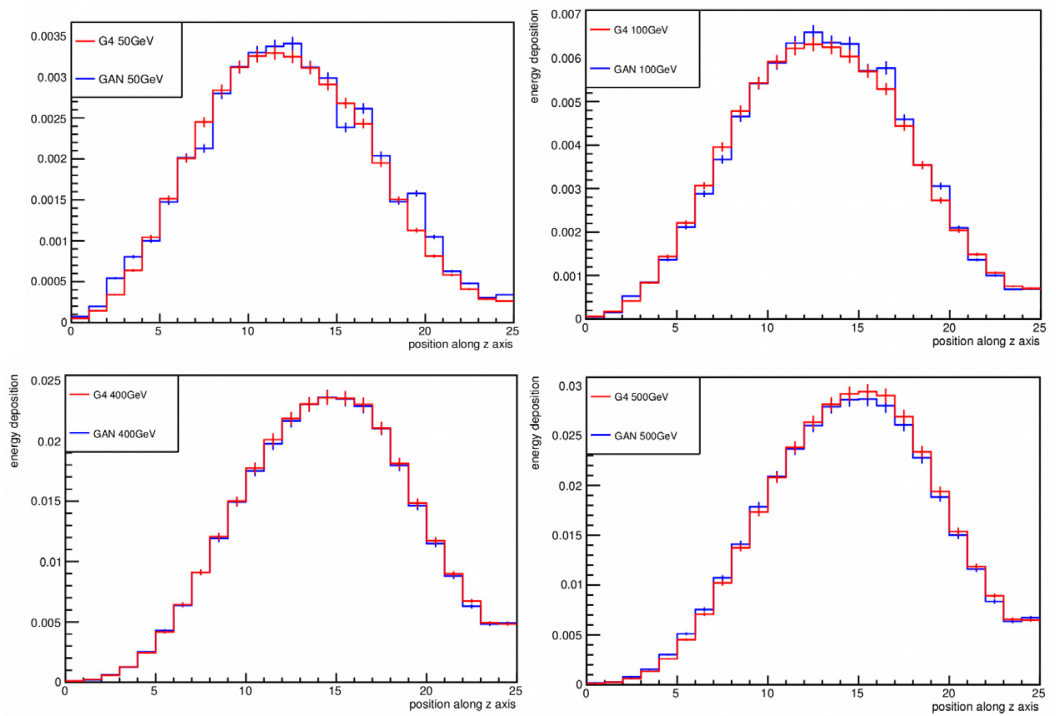

Figure 4. Longitudinal energy profiles along $\mathrm{z}$ for 400 and $500 \mathrm{GeV}$ electrons. Geant 4 prediction is shown in blue and the GAN's in red. Units are GeV/50.
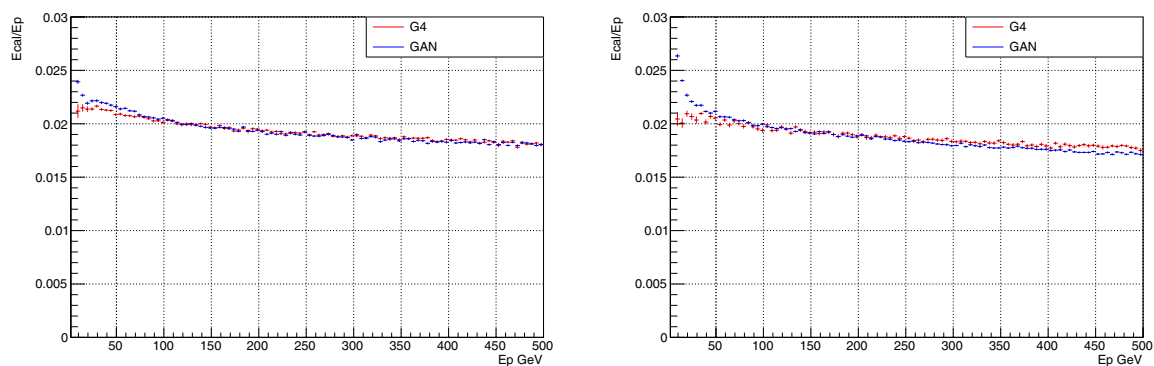

Figure 5. Calorimeter sampling fraction as a function of the incoming electron (left) and neutral pion (right) energy as predicted by Geant 4 simulation (in blue) and GANs (in red)

We have trained the network using 200000 single electrons generated with energies sampled from a uniform $[100-200] \mathrm{GeV}$ spectrum and an angle $\theta$ sampled from a $\left[60^{\circ}-120^{\circ}\right]$ spectrum . Figure 6 compares the energy shower shapes generated by the angle-aware 3DGAN to the Geant4 showers. Although preliminary, these results show very good agreement to Monte Carlo. Detailed validation studies are on-going. This results were obtained by manually adjusting architecture hyper-parameters to fit the new image size. Our plan is to test an automated optimisation procedure to achieve similar or better performance and extend to different calorimeter examples.

\section{Computing performance and training time}

Once trained, the GAN simulation tool is a relatively lightweight application: a few MB are enough to describe the layers configuration for the two networks and the inference step is 

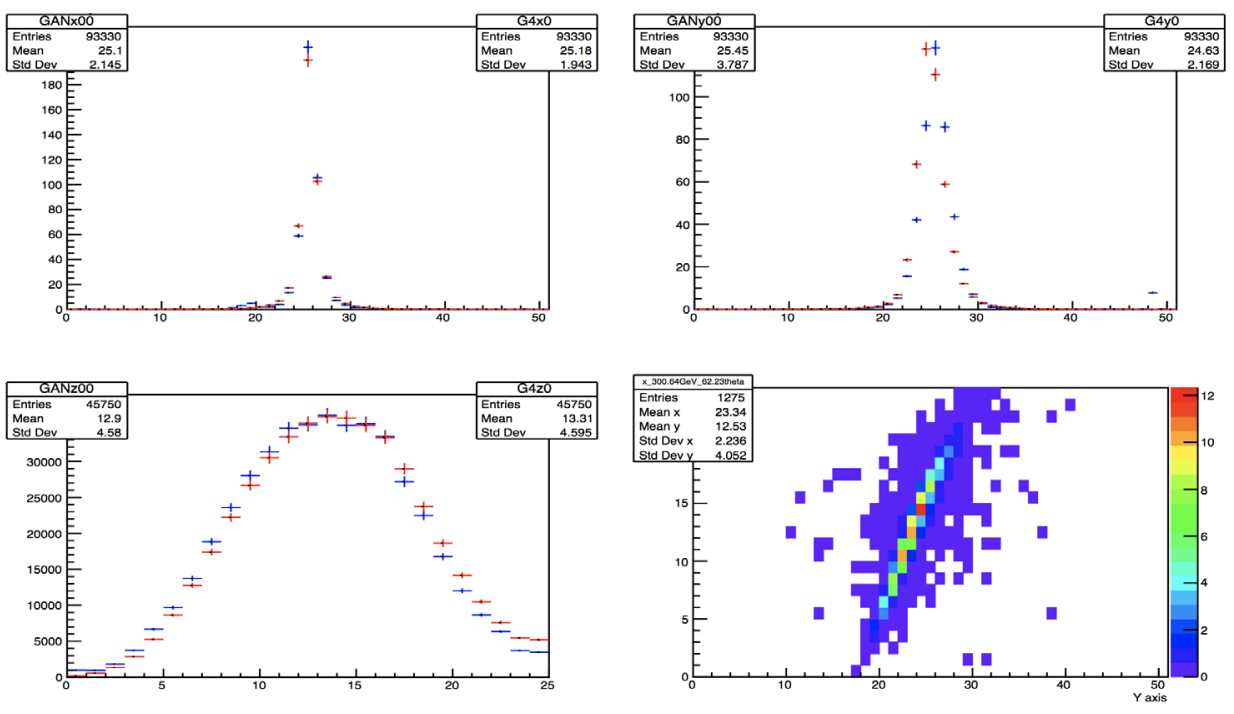

Figure 6. Top: Transverse energy profile along x (left) and y (right) for 100-200 GeV electrons, with incident angles uniformly distributed between $60 \mathrm{deg}$ and $120 \mathrm{deg}$. Bottom: Longitudinal energy profile along $\mathrm{z}$ for $100-200 \mathrm{GeV}$ electrons, with incident angles uniformly distributed between $60 \mathrm{deg}$ and $120 \mathrm{deg}$ (left). Example $Y-Z$ view of an electron shower with energy $=150 \mathrm{GeV}$ and $\theta=60 \mathrm{deg}$ (right). Geant4 simulation is in red. GAN results in blue.

orders of magnitude faster than a standard Monte Carlo. We have run a test on a Intel Xeon 8180 processor (codenamed "Skylake") measuring the time it takes to generate one electromagnetic shower for our benchmark detector. We obtained 17 s/shower using Geant4 and 4 $\mathrm{ms} /$ shower using our trained GAN model, yielding a speedup factor larger than 2500. Using dedicated hardware, such as GPGPUs, the generation time reduces further (we choose not to quote comparison results since the Geant4 application can not run on GPGPUs). On the other hand, the GAN training process is more time consuming. As mentioned above, the core structure of both generator and discriminator is based on four 3-dimensional convolutional layers, so the models are not very deep, compared to other existing convolutional networks: in total, the generator and discriminator networks sum up to about $1 \mathrm{M}$ parameters, when a relatively small latent space is chosen (200 elements). Moreover, the adversarial training itself, relying on continuous feedback between the discriminator and the generator is particularly time consuming. We also train each network twice following a process that is explained in [10]: in fact, a two-step discriminator training (on separate real and fake image batches) helps to insure fast convergence of the network [14]. This results in a relatively large computing time: training the 3DGAN on 200,000 electrons for 30 epochs takes about 1 day on a NVIDIA P100: training time on the angle dataset (images are $4 \mathrm{x}$ larger) almost doubles. While this does not represent, per se, a problem, it critically impacts any attempt to run hyper-parameter scans or implement meta-optimization algorithms, which are key to our model generalisation effort. For this reason we have dedicated some efforts in the study and benchmarking of different approaches for parallel training of 3DGAN on distributed systems, both on HPC systems [15-17] and cloud, in the context of the HNSciCloud project [18] . 


\section{Conclusion and future plans}

Monte Carlo production has been so far a major fraction of the LHC Grid computing workload and the High Luminosity LHC experiments needs will increase by orders of magnitude. In this context, generative models relying on the possibility to treat detector response as images, seem natural candidates to speedup simulation. Generative Adversarial Networks, in particular, require relatively small amounts of data to train and are the subject of many ongoing studies. Their performance as imaging tools for calorimeter simulation is very promising, and from a computing resources perspective, the gain in the time needed to generate a shower is huge. As mentioned above it reduces to roughly $4 \mathrm{~ms} /$ shower compared to the $17 \mathrm{~s} /$ shower needed by Geant 4 simulation on a Intel Xeon 8080 CPU. We have presented an application of 3D convolutional GANs to the simulation of high-granularity detectors. We have successfully generated three-dimensional images of energy showers, according to different energy and incident angle values. A detailed validation of the physics results based on the comparison to state-of-the-art Monte Carlo software shows very good agreement. We plan to extend this performance studies to the angle-aware architecture and experiment different optimisation frameworks in order to adapt the best architecture design to the specific simulation use-case. Furthermore, we will test the performance of our approach on different high granularity calorimeter architectures such as CLIC hadronic calorimeter [7] or the SDHCAL prototype [19].

\section{Acknowledgement}

The authors wish to acknowledge the contribution of Intel to the project through the Intel Performance Computing Centre (IPCC) program.

\section{References}

[1] Bird I 2016 WLCG wokshop

[2] Goodfellow I et al. 2014 (Preprints stat-ml/1406.2661)

[3] Karras et al., 2018 (ICLR2018)

[4] $\mathrm{Wu} \mathrm{J}$ et al 2016. NIPS conf proc.

[5] Paganini M et al 2017 (Preprint hep-ex/1701.05927)

[6] Paganini M et al 2017 (Preprint hep-ex/1705.02355)

[7] Tehrani, N et al 2017 (Preprint CERN/CLICdp-Note-2017-001)

[8] Geant4 project, "Geant4" [software], version 10.3.2, 2017. Available from https://github.com/Geant4/geant4/releases/tag/v10.3.2 [accessed 2017-08-17]

[9] Pierini M. 2016 DS@HEP at the Simons Foundation

[10] Vallecorsa S et al., ACAT2017 proceedings, Journal of Physics: Conference Series, 1085, 3, 2018.

[11] Mirza M, Osindero S 2014 (Preprints abs/1411.1784)

[12] Odean A et al., (Preprints stat.ML/1610.09585)

[13] Hinton $\mathrm{G}$ et al., Lecture 6a overview of minibatch gradient descent, 2012.

[14] Chintala S et al., https://github.com/soumith/ganhacks.

[15] Vallecorsa S et al., ISC 2018 Workshops, LNCS 11203, 2018.

[16] Vlimant, J-R et al. CHEP2018 proceedings.

[17] Vallecorsa S et a., HiPC 2018 proceedings.

[18] https://www.hnscicloud.eu.

[19] Laktineh I et al., 2016 (Preprint hep-ex/1602.02276v2) 